\title{
Terahertz Vortex Beam as a Spectroscopic Probe of Magnetic Excitations
}

\author{
A. A. Sirenko, ${ }^{1, *}$ P. Marsik, ${ }^{2}$ C. Bernhard, ${ }^{2}$ T. N. Stanislavchuk, ${ }^{1}$ V. Kiryukhin, ${ }^{3}$ and S.-W. Cheong ${ }^{3}$ \\ ${ }^{1}$ Department of Physics, New Jersey Institute of Technology, Newark, New Jersey 07102, USA \\ ${ }^{2}$ Department of Physics, University of Fribourg, CH-1700 Fribourg, Switzerland \\ ${ }^{3}$ Rutgers Center for Emergent Materials and Department of Physics and Astronomy, Rutgers University, \\ Piscataway, New Jersey 08854, USA
}

(Received 20 February 2019; published 12 June 2019)

\begin{abstract}
Circularly polarized light with spin angular momentum is one of the most valuable probes of magnetism. We demonstrate that light beams with orbital angular momentum (OAM), or vortex beams, can also couple to magnetism exhibiting dichroisms in a magnetized medium. Resonant optical absorption in a ferrimagnetic crystal depends strongly on both the handedness of the vortex and the direction of the beam propagation with respect to the sample magnetization. This effect exceeds the conventional dichroism for circularly polarized light. Our results demonstrate the high potential of the vortex beams with OAM as a new spectroscopic probe of magnetism in matter.
\end{abstract}

DOI: 10.1103/PhysRevLett.122.237401

Vortex beams of photons [1-3] and electrons [4,5] can carry orbital angular momentum (OAM) in addition to spin angular momentum (SAM); the latter is known in optics as the circular polarization of light. Although applications of the optical vortex beams have been demonstrated previously in quantum communication [6,7], astronomy [8], and optical tweezers [9-11], they have not been applied for spectroscopy of magnetic excitations yet. Despite the intuitive expectation that vortex beams can instantly reveal new phenomena in condensed matter systems, there was a consensus that new effects can be expected only in scenarios beyond the electric dipole approximation [12-14]. Here we present experimental results for interaction of the optical vortex beams with nonlocal magnetic excitations that are similar to spin waves.

Optical vortices can be produced by different methods for manipulation of the light phase and polarization $[15,16]$. Here we implemented a custom-designed transparent axicon optics that allows formation of vortices in a broadband terahertz spectral range. Vortex production became possible due to the transverse coherence of the terahertz source [17-20]. Terahertz beams are important for future applications in magnonics, i.e., for signal processing based on light manipulation of the traveling spin waves in magnetic device structures [21]. In our experiments, we addressed two related question about dichroism and nonreciprocity of the terahertz vortex beam propagation in a magnetized medium. The general interest in nonreciprocal optical effects is based on the possibility of verifying fundamental principles of symmetry and revealing details for new interactions, such as the dynamic magnetoelectric effect in magnetically ordered crystals [22-26].

The time domain terahertz optical setup consisted of a terahertz photoconductive antenna emitter and detector, along with wire-grid linear polarizers and an optical retarder [27]. The coherent terahertz beam was focused on the sample with the $f$ number equal to 10 using a $50 \mathrm{~mm}$ off-axis parabolic mirror. The spectral range was between 0.11 and $1.65 \mathrm{THz}$, or between 3 and $55 \mathrm{~cm}^{-1}$. A single Fresnel prism (FP) made of TOPAS was used as a broadband optical retarder to convert from linear $\left(\vec{e}_{x} \pm \vec{e}_{y}\right)$ to the right- and left-hand circular polarizations $\vec{e}_{R}=\vec{e}_{x}-i \cdot \vec{e}_{y}$ and $\vec{e}_{L}=\vec{e}_{x}+i \cdot \vec{e}_{y}$. An axicon retarder made of transparent silicon was used to produce broadband terahertz vortex beams with two orthogonal directions of electric field structured around the beam propagation vector $\vec{k}$. Figure 1 shows a linearly polarized terahertz beam (from the right) slowly focused towards the sample (on the left). After the FP retarder, the circularly polarized light passes through a four-bounce axicon retarder. The sign of the

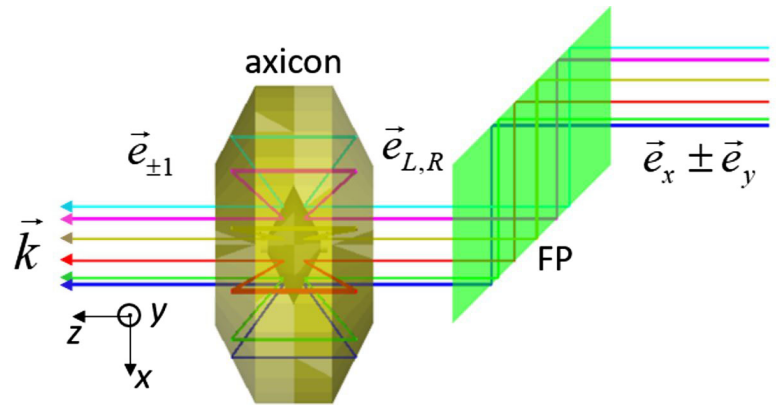

FIG. 1. Linearly polarized terahertz radiation is converted into vortex beam modes $\vec{e}_{ \pm 1}$ using a combination of a Fresnel prism (FP) and an axicon. After passing through a two-bounce FP retarder, the linearly polarized light $\vec{e}_{x} \pm \vec{e}_{y}$ becomes circularly polarized $\vec{e}_{L, R}$. After passing through the axicon, the beam acquires a vortex phase $\vec{e}_{ \pm 1}$ while losing its circular polarization. 

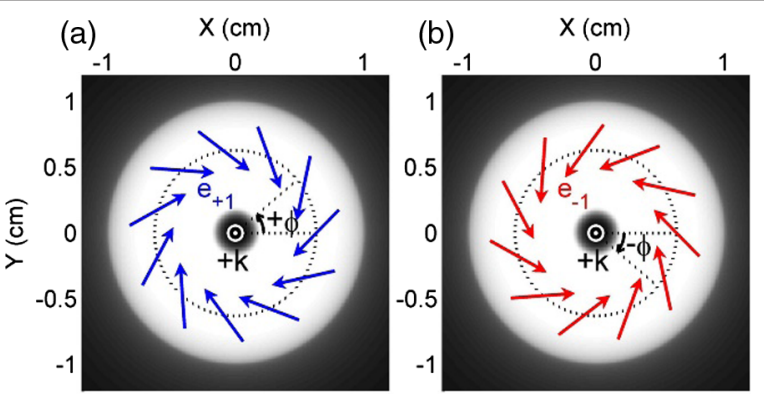

\begin{abstract}
II
\end{abstract}

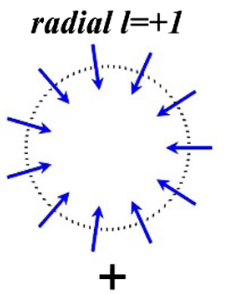

azimuthal $l=+1$

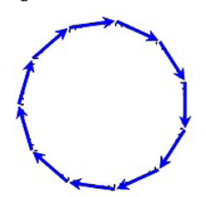

II

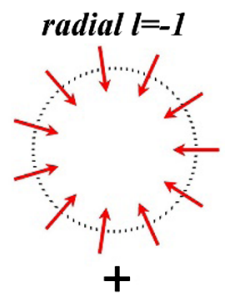

azimuthal $l=-1$
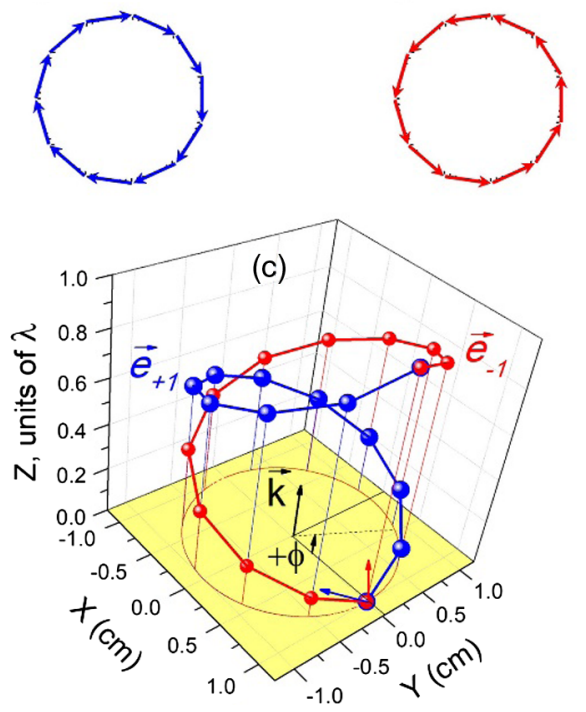

FIG. 2. Calculated radially independent electric fields and equal phase trajectories in the vortex beams. (a),(b) Projections of electric field for the $\vec{e}_{+1}$ and $\vec{e}_{-1}$ modes are shown in the $x-y$ plane. The white areas represent the intensity distribution of the vortex beam. The beam propagation direction $\vec{k}$ is along the positive $z$ axis. Decoupling of the vortex modes into the azimuthal and radial ones is also shown. (c) Spatial variation of the equal phase trajectories. $\vec{e}_{+1}$ and $\vec{e}_{-1}$ form the right-hand and left-hand spirals. The corresponding electric field vectors in the $x$-y plane at $z=0$ and $\phi=0$ are shown with red and blue arrows.

output vortex beams $\vec{e}_{ \pm 1}$ is determined by the input circular polarization: $\vec{e}_{L} \rightarrow \vec{e}_{+1}$ and $\vec{e}_{R} \rightarrow \vec{e}_{-1}$. The polarization conversion occurs due to phase changes during the four internal reflections inside the axicon.

The electric field profiles in two vortex beams $\vec{e}_{+1}$ and $\vec{e}_{-1}$ are shown in Fig. 2. The azimuthal dependence of $\vec{e}_{l}$ is $\vec{e}_{l}(\vec{r}, \phi) \approx(\vec{r} / r) \cdot \exp \left[i \cdot l\left(\phi-\phi_{0}\right)\right]$, where $\phi$ is the vortex phase, the initial phase is $\phi_{0}=3 \pi / 4, \vec{r}$ is the radial coordinate, and $l$ is the topological number. $l= \pm 1$ means that the electric field phase changes by $\pm 2 \pi$ for one complete rotation around the beam axis [28]. $\vec{e}_{+1}$ and $\vec{e}_{-1}$ have nearly orthogonal directions of the electric field for each ray with the same $(x, y)$ coordinates [Figs. 2(a) and 2(b)]. Their equal phase surfaces make right-hand and left-hand spirals around the $\vec{k}$ vector for $\vec{e}_{+1}$ and $\vec{e}_{-1}$ [Fig. 2(c)]. Given the transverse coherence of the terahertz source, $l= \pm 1$ defines the sign of the OAM for the whole beam with $L=l \cdot \hbar$ per photon.

For our experiments with the broadband terahertz vortex beams, we were looking for a system with collective magnetic excitations in a transparent medium that can be magnetized at room temperature. Rare earth $(R)$ iron garnets ( $R$-IG) with four formula units of $R_{3} \mathrm{Fe}_{5} \mathrm{O}_{12}$ satisfy these requirements $[29,30]$. Interesting magneto-optical and magnetostriction effects in $R$-IG are related to the ferrimagnetic order in the $\mathrm{Fe}$ spin sublattice with $T_{N}=550 \mathrm{~K}$, and to the anisotropic superexchange interaction between $\mathrm{Fe}^{3+}$ and $R^{3+}$ spins [31,32]. Discovery of magnetoelectric and magnetodielectric effects in Tb-IG at low magnetic fields of less than $0.2 \mathrm{~T}$ renewed the interest in $R$-IGs [33]. Formation of the local electric polarization is induced by magnetic ordering in Tb-IG [34] and antiferroelectric (AFE) ordering in Dy-IG occurs in the same lowtemperature range as the magnetic ordering of $\mathrm{Dy}^{3+}$ spins at $T<T_{C}=16 \mathrm{~K}$ [35]. At low temperatures, garnets have several nonlocal magnetic excitations, such as ligand field (LF) and Kaplan-Kittel (KK) modes [36-38]. These modes are of magnetic origin produced by the mutual precession of the $R^{3+}$ and $\mathrm{Fe}^{3+}$ spins. The experimental temperature dependencies of the LF and KK excitations in Dy-IG (Fig. 3) are similar to that for antiferromagnetic resonances, or magnons at $\vec{k}=0$, in the magnetically ordered system with several interacting spins [39-41]. Interaction of the LF and KK modes in Dy-IG with vortex optical beams is the main focus of our experiments. More details for magnetic and optical properties of Dy-IG are in the Supplemental Material [42].

The high-temperature flux growth technique was utilized to produce single crystals of Dy-IG. The same sample with a platelike shape with the 111 crystallographic orientation, with a thickness of about $1 \mathrm{~mm}$, and in-plane dimensions of about $7 \times 8 \mathrm{~mm}^{2}$ was used for transmission experiments using both circular polarized light and vortex beams. Before each measurement, the sample was magnetized normal to the sample surface using $\mathrm{Nd}$ magnets, which produced a field of $0.6 \mathrm{~T}$ in the sample. Two transmission spectra for $\vec{e}_{R}$ and $\vec{e}_{L}$ measured at $T=8 \mathrm{~K}$ are shown in Fig. 4(a). They are dominated by the strongest LF transition at $13 \mathrm{~cm}^{-1}$ that is nearly saturated at low temperatures. Several additional weaker lines at 23, 28, and $44 \mathrm{~cm}^{-1}$ are also clearly resolved. Two lines at 17 and $28 \mathrm{~cm}^{-1}$, 


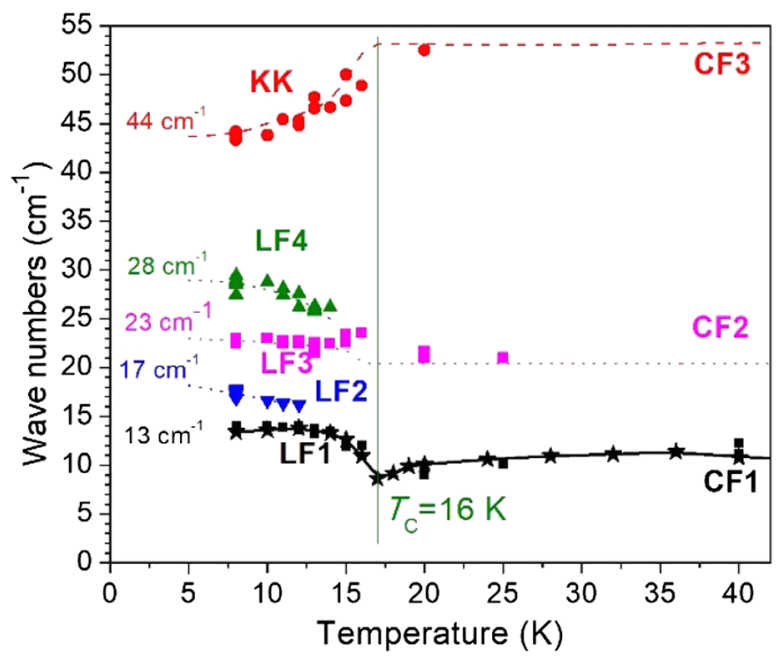

FIG. 3. Energies of the LF, KK, and CF modes vs temperature. Experimental data for energies of the LF, KK, and CF modes are shown with solid symbols. Data from Ref. [39] obtained with conventional linearly polarized light are shown with dashed curves for comparison. The solid black curve is a guide for the eye.

measured for magnetization direction $\vec{M}$ being antiparallel $\vec{k}$, appear stronger in $\vec{e}_{L}$ compared to that for $\vec{e}_{R}$. We observed a reversal of the absorption selection rules after the sample (and its magnetization $\vec{M}$ ) was rotated by $180^{\circ}$ with respect to the vertical laboratory axis $y$, i.e., after the $\hat{R}_{y, 180^{\circ}}$ operation.

The same magnetized sample was measured using vortex beams. The corresponding spectra for different temperatures are shown in Figs. 4(b)-4(g), with the magnetization of the sample being parallel [Figs. 4(b)-4(d)] and antiparallel [Figs. 4(e)-4(g)] with respect to the beam propagation direction. The transmission spectra were fitted using a simple harmonic oscillator model. The oscillator strengths were determined as in Ref. [35] using the normalized units of dielectric permittivity and magnetic permeability, $\varepsilon_{\infty}$ and $\mu_{\infty}$ (see the Supplemental Material [42] for details). At $T<T_{C}=16 \mathrm{~K}$, we observe significant differences in the oscillator strength of the LF modes at 17, 23, and $28 \mathrm{~cm}^{-1}$ between $\vec{e}_{+1}$ and $\vec{e}_{-1}$ [Fig. 4(b)]. The combined oscillator strengths for the modes at 17,23 , and $28 \mathrm{~cm}^{-1}$ averaged for three lowest temperatures, all below $T_{C}=16 \mathrm{~K}$, are $S_{T,-1}=0.14$ and $S_{T,+1}=0.09$. The corresponding vortex polarization for the oscillator strength $\rho_{ \pm 1}=\left(S_{T,+1}-S_{T,-1}\right) /\left(S_{T,+1}+S_{T,-1}\right)$ amounts to $-22 \%$. Above $T_{C}=16 \mathrm{~K}$, the two LF excitations at 23 and $28 \mathrm{~cm}^{-1}$ merge into a single line at $21 \mathrm{~cm}^{-1}$ that remains at the same energy until it disappears at high temperatures [Figs. 4(c) and 4(d)]. This is a result of the thermal repopulation of the crystal field (CF) levels of $\mathrm{Dy}^{3+}$ for $T>50 \mathrm{~K}$. At high temperatures around $40 \mathrm{~K}$, one can still see that the lowest energy mode at $13 \mathrm{~cm}^{-1}$ also
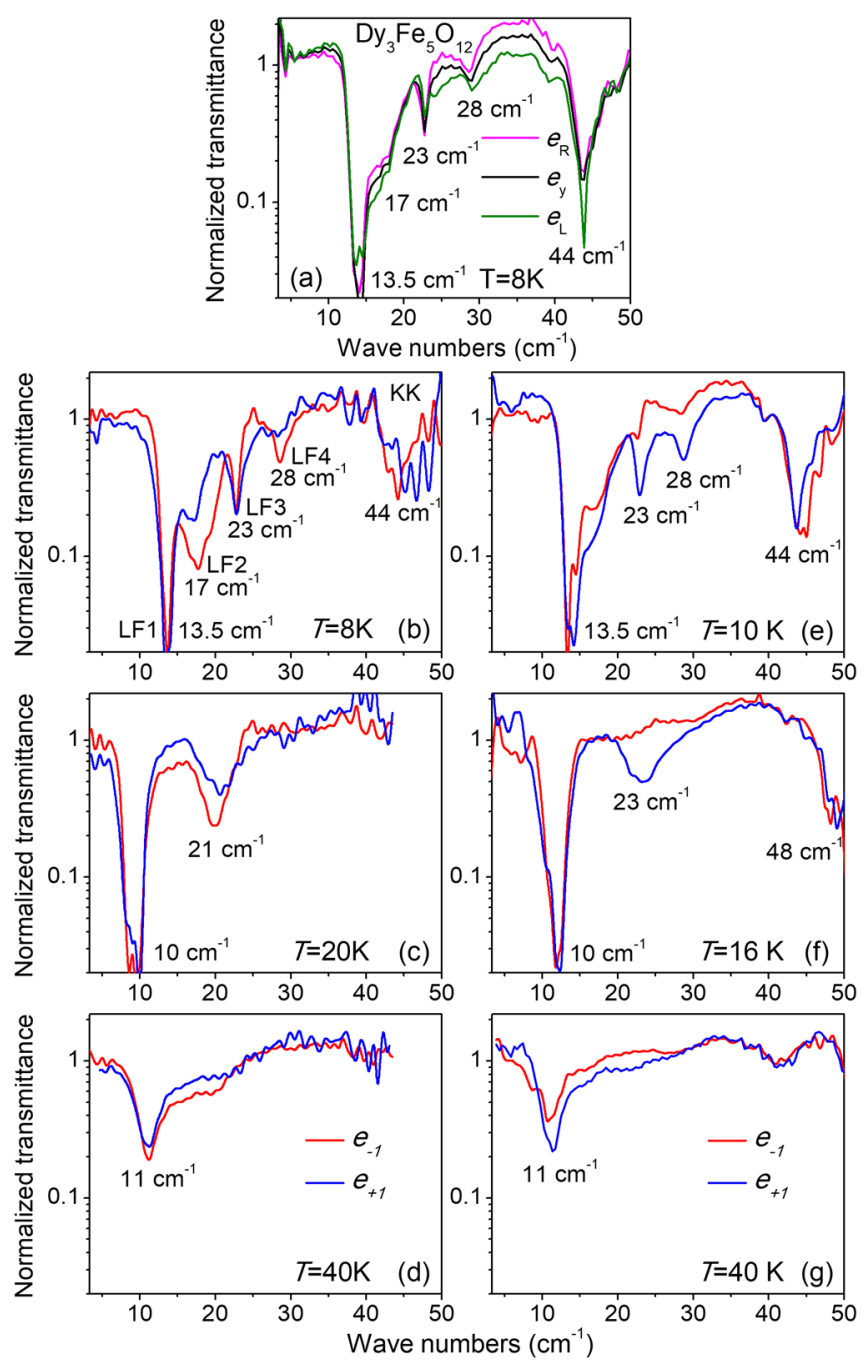

FIG. 4. Magnetic dichroism in transmittance spectra for circularly polarized light and vortex beams. (a) Normalized transmittance spectra for circularly polarized light $\vec{e}_{R}$ and $\vec{e}_{L}$, and for conventional linearly polarized light $\vec{e}_{y}$. The magnetization vector $\vec{M}$ is antiparallel to $\vec{k}$. (b)-(d) Normalized transmittance spectra for three temperatures and two orthogonal vortex beams $\vec{e}_{+1}$ (blue spectra) and $\vec{e}_{-1}$ (red spectra) measured for $\left\{\vec{k}, \vec{e}_{ \pm 1}, \vec{M}\right\}$ with the magnetization vector $\vec{M}$ parallel to $\vec{k}$. (e)-(g) The same for the opposite directions of the light propagation with respect to the sample: $\left\{\vec{k}, \vec{e}_{ \pm 1}, \hat{R}_{y, 180^{\circ}}(\vec{M})\right\}$, with the magnetization vector $\vec{M}$ antiparallel to $\vec{k}$. All experimental data in (a)-(g) are normalized to that measured at $T=75 \mathrm{~K}$.

reveals some weak dichroism for $\vec{e}_{+1}$ and $\vec{e}_{-1}$. After the sample rotation $\hat{R}_{y, 180^{\circ}}$, we observed that the selection rules for the vortex beam absorption reversed, and the stronger peaks in $\vec{e}_{-1}$ become weaker than that for $\vec{e}_{+1}$ [Figs. 4(e)-4(g)]. The rotation $\hat{R}_{y, 180^{\circ}}$ was repeated twice, and reproducibility of the switching of the preferable polarization for the modes has been confirmed. For the low-temperature spectra shown in Fig. 4(e), we obtained 
$S_{T,-1}=0.11$ and $S_{T,+1}=0.13$ with the corresponding polarization $\rho_{ \pm 1}=+8.3 \%$.

The selection rules for the LF modes depend strongly on the combination of experimental parameters for both circularly polarized light and the vortex beam propagating through the magnetized crystal. The observed dichroic effect for the circularly polarized light in Fig. 4(a) can be quantified in terms of the oscillator strength polarization $\rho_{R, L}=\left(S_{T, L}-S_{T, R}\right) /\left(S_{T, R}+S_{T, L}\right)$, which amounts to about $\pm 3 \%$. It represents the conventional circular dichroism due to the coupling between the SAM of the photons and magnetization of the medium. In contrast, the observed vortex dichroism for the beams with opposite OAM ( $L=l \cdot \hbar$ with $l= \pm 1$ ) is a new effect and, thus, requires a detailed discussion. Figure 4(b) shows that the two different combinations of the light propagation direction, the sign of vorticity, and the magnetization direction of the $\mathrm{Fe}^{3+}-\mathrm{Dy}^{3+}$ system, $\left\{\vec{k}, \vec{e}_{+1}, \vec{M}\right\}$ and $\left\{\vec{k}, \vec{e}_{-1}, \vec{M}\right\}$, give rise to different oscillator strengths for the LF modes. Notably, these differences are even stronger than that for the circular dichroism in Fig. 4(a). The vortex dichroism for $\vec{e}_{+1}$ and $\vec{e}_{-1}$ can be understood in terms of the symmetry arguments sketched in Fig. 5(a). Note that there is no sequence of symmetry elements, such as inversion, mirror reflection, or rotation, that would transform $\left\{\vec{k}, \vec{e}_{+1}, \vec{M}\right\}$ into

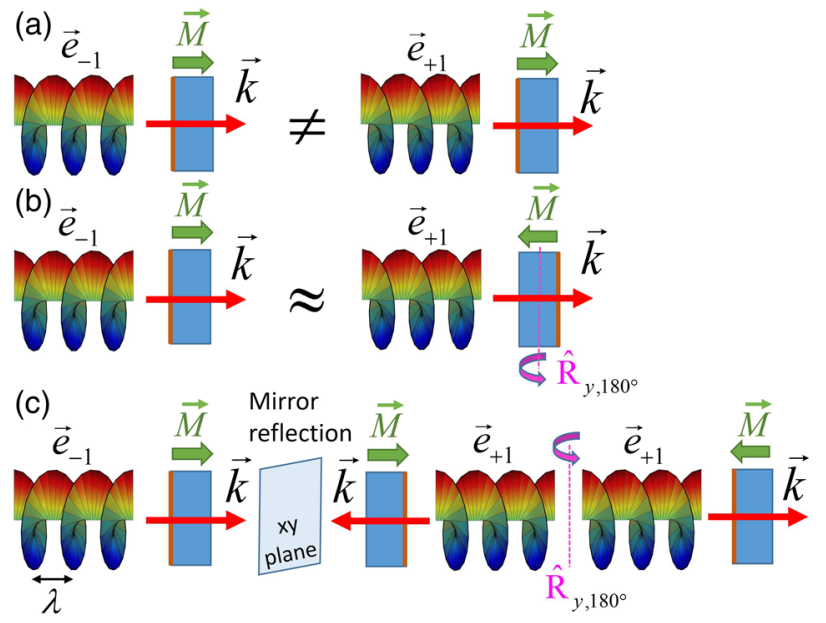

FIG. 5. Schematics of the observed dichroic effects. Propagation of the wave front in the vortex beam is illustrated with color rendering. The closest distance between the same colors along the $z$ direction corresponds to the wavelength of light $\lambda$. (a) Vortex beam dichroism: $\left\{\vec{k}, \vec{e}_{-1}, \vec{M}\right\} \neq\left\{\vec{k}, \vec{e}_{+1}, \vec{M}\right\}$. (b) The same for the observed inversion of the selection rules for rotation of the magnetized sample that also resulted in the sign change for $\rho_{ \pm 1}$. (c) Transformation between $\left\{\vec{k}, \vec{e}_{-1}, \vec{M}\right\}$ and $\left\{\vec{k}, \vec{e}_{+1},-\vec{M}\right\}$ can be obtained by applying a mirror reflection that is perpendicular to $z$ and rotation around the $y$ axis, both for the whole experimental setup. The sample is shown with blue rectangles with green arrows for the sample magnetization direction $\vec{M}$. One of the sample faces is marked with a vertical brown line. $\left\{\vec{k}, \vec{e}_{-1}, \vec{M}\right\}$. Thus, symmetry allows for the observed vortex dichroism.

The difference between the $\vec{e}_{+1}$ and $\vec{e}_{-1}$ modes for the magnetized sample can be better illustrated if one decouples each mode into coherent combinations of azimuthal and radial modes $\vec{e}_{l}(\vec{r}, \phi) \approx(\vec{r} / r) \cdot\left[\exp \left[i \cdot l\left(\phi-90^{\circ}\right)\right]-\right.$ $\exp [i \cdot l \cdot \phi]]$ [Figs. 2(a) and 2(b)]. In this representation, the second terms for the radial component $-\exp [i \cdot l \cdot \phi]$ are similar for both $\vec{e}_{+1}$ and $\vec{e}_{-1}$, while the azimuthal components $\exp [i \cdot l \cdot(\phi-90)]$ correspond to two opposite circulations of the electric fields around the beam axis. The azimuthal components resemble circular currents that produce magnetic fields directed along or opposite to $\vec{k}$, which can modulate the sample magnetization $\vec{M}$, making the $\vec{e}_{+1}$ and $\vec{e}_{-1}$ beams nonequivalent with respect to $\vec{k}$ and $\vec{M}$. Such symmetry arguments can help with the qualitative interpretation of the observed dichroism. The measured oscillator strength polarization $\rho_{ \pm 1}$ allowed us to quantify the effect.

Symmetry arguments can also help us to explain the observed inversion of the selection rules for the two vortex beams when the magnetized sample is rotated by $180^{\circ}$ around the $y$ axis $\left\{\vec{k}, \vec{e}_{ \pm 1}, \vec{M}\right\} \approx\left\{\vec{k}, \vec{e}_{\mp 1}, \hat{R}_{y, 180^{\circ}}(\vec{M})\right\}$, as shown in Fig. 5(b). Indeed, the set $\left\{\vec{k}, \vec{e}_{+1}, \vec{M}\right\}$ can be transformed into $\left\{\vec{k}, \vec{e}_{-1},-\vec{M}\right\}$ by applying both a mirror reflection with the plane normal to $z$ and rotation $\hat{R}_{y, 180^{\circ}}$ [Fig. 5(c)]. The handedness of the azimuthal components $\vec{e}_{ \pm 1}$ transforms by the mirror reflection keeping the $\vec{M}$ direction unchanged. The rotation $\hat{R}_{y, 180^{\circ}}$ changes the sign of magnetization $\vec{M}$ preserving the handedness of the vortex. Thus, the vortex mode should also be inverted to achieve the same experimental conditions for the opposite magnetization. These arguments support our observation of the similar selection rules for $\left\{\vec{k}, \vec{e}_{ \pm 1}, \vec{M}\right\}$ and $\left\{\vec{k}, \vec{e}_{\mp 1}, \hat{R}_{y, 180^{\circ}}(\vec{M})\right\}$, which can be seen in Figs. $4(\mathrm{~b})-4(\mathrm{~g})$.

The sample rotation with respect to the terahertz beam $\hat{R}_{y, 180^{\circ}}$ represents a test for the reciprocity of the light propagation with $\pm \vec{k}$. The observed difference between the absolute values for $\rho_{ \pm 1}$, which are $|-22 \%|$ and $|+8.3 \%|$ for the data before and after the sample rotation $\hat{R}_{y, 180^{\circ}}$ in Figs. 4(b) and 4(e), corresponds to the directional dichroism of the vortex beams. For example, the intensity of the LF mode at $17 \mathrm{~cm}^{-1}$ is significantly different for the two directions of the light propagation. This difference could be explained by the lack of a center of inversion for the $\mathrm{Dy}^{3+}$ sites and, plausibly, by the AFE ordering at low temperatures.

In conclusion, the terahertz vortex beams with opposite OAM with $l= \pm 1$ were generated using transparent axicons. The observed vortex beam dichroism in magnetized Dy-IG is the most pronounced in resonance with the LF modes of $\mathrm{Dy}^{3+}$. The magnitude of dichroism for the 
vortex beams, expressed in terms of the oscillator strengths of the modes, is stronger than that for circularly polarized light. Application of the light beams with both OAM and SAM can be useful in the future studies of the spin and orbital contributions to magnetism. The directional dichroism for vortex beams may also have potential applications for studies of collective excitations in magnetic solids.

The authors are grateful to Tao Zhou, D. Talbayev, and G. L. Carr for the useful discussions and to T. Cyrulik for help with the modeling of the light propagation. Work at the New Jersey Institute of Technology and Rutgers University was supported by the U.S. DOE under Contract No. DEFG02-07ER46382. The work at the University of Fribourg was funded by the Schweizer Nationalfonds (SNF) by Grant No. 200020-172611.

*Corresponding author. sirenko@njit.edu

[1] L. Allen, M. W. Beijersbergen, R. J. C. Spreeuw, and J. P. Woerdman, Phys. Rev. A 45, 8185 (1992).

[2] G. Molina-Terriza, J. P. Torres, and L. Torner, Nat. Phys. 3, 305 (2007).

[3] Q. Zhan, Cylindrical Vector Beams in Vectorial Optical Fields (World Scientific Publishing, Singapore, 2014).

[4] J. Yuan, S. M. Lloyd, and M. Babiker, Phys. Rev. A 88, 031801(R) (2013).

[5] J. Verbeeck, H. Tian, and P. Schattschneider, Nature (London) 467, 301 (2010).

[6] J. Wang, J.-Y. Yang, I. M. Fazal, N. Ahmed, Y. Yan, H. Huang, Y. Ren, Y. Yue, S. Dolinar, M. Tur, and A. E. Willner, Nat. Photonics 6, 488 (2012).

[7] A. Mair, A. Vaziri, G. Weihs, and A. Zeilinger, Nature (London) 412, 313 (2001).

[8] M. Harwit, Astrophys. J. 597, 1266 (2003).

[9] H. He, M. E. J. Friese, N. R. Heckenberg, and H. RubinszteinDunlop, Phys. Rev. Lett. 75, 826 (1995).

[10] A. T. O’Neil, I. MacVicar, L. Allen, and M. J. Padgett, Phys. Rev. Lett. 88, 053601 (2002).

[11] J. E. Curtis and D. G. Grier, Phys. Rev. Lett. 90, 133901 (2003).

[12] M. Babiker, C. R. Bennett, D. L. Andrews, and L. C. Davila Romero, Phys. Rev. Lett. 89, 143601 (2002).

[13] F. Giammanco, A. Perona, P. Marsili, F. Conti, F. Fidecaro, S. Gozzini, and A. Lucchesini, Opt. Lett. 42, 219 (2017).

[14] C. T. Schmiegelow, J. Schulz, H. Kaufmann, T. Ruster, U. G. Poschinger, and F. Schmidt-Kaler, Nat. Commun. 7, 12998 (2016).

[15] S. Franke-Arnold and N. Radwell, Opt. Photonics News 28, 28 (2017).

[16] S. S. R. Oemrawsingh, E. R. Eliel, J. P. Woerdman, E. J. K. Verstegen, J. G. Kloosterboer, and G. W. 't Hooft, J. Opt. A 6, S288 (2004).

[17] Z. Xie, X. Wang, J. Ye, S. Feng, W. Sun, T. Akalin, and Y. Zhang, Sci. Rep. 3, 3347 (2013).

[18] R. Imai, N. Kanda, T. Higuchi, K. Konishi, and M. Kuwata-Gonokami, Opt. Lett. 39, 3714 (2014).
[19] T. Arikawa, S. Morimoto, and K. Tanaka, Opt. Express 25, 13728 (2017)

[20] Q. Lin, S. Zheng, Q. Song, X. Zeng, Y. Cai, Y. Li, Z. Chen, L. Zha, X. Pan, and S. Xu, Opt. Lett. 44, 887 (2019).

[21] A. V. Chumak, V. I. Vasyuchka, A. A. Serga, and B. Hillebrands, Nat. Phys. 11, 453 (2015).

[22] I. Kézsmárki, N. Kida, H. Murakawa, S. Bordács, Y. Onose, and Y. Tokura, Phys. Rev. Lett. 106, 057403 (2011).

[23] S. Bordács, I. Kézsmárki, D. Szaller, L. Demko, N. Kida, H. Murakawa, Y. Onose, R. Shimano, T. Rõõm, U. Nagel, S. Miyahara, N. Furukawa, and Y. Tokura, Nat. Phys. 8, 734 (2012).

[24] I. Kézsmárki, U. Nagel, S. Bordács, R. S. Fishman, J. H. Lee, H. T. Yi, S.-W. Cheong, and T. Rõõm, Phys. Rev. Lett. 115, 127203 (2015).

[25] S. Yu, B. Gao, J. W. Kim, S.-W. Cheong, M. K. L. Man, J. Madéo, K. M. Dani, and D. Talbayev, Phys. Rev. Lett. 120, 037601 (2018).

[26] S.-W. Cheong, D. Talbayev, V. Kiryukhin, and A. Saxena, npj Quantum Mater. 3, 19 (2018).

[27] P. Marsik, K. Sen, J. Khmaladze, M. Yazdi-Rizi, B. P. P. Mallett, and C. Bernhard, Appl. Phys. Lett. 108, 052901 (2016).

[28] E. J. Galves, in The Angular Momentum of Light, edited by D. Andrews and M. Babiker (Cambridge University Press, Cambridge, England, 2013).

[29] F. Sayetat, J. X. Boucherle, and F. Tcheou, J. Magn. Magn. Mater. 46, 219 (1984).

[30] R. Hock, H. Fuess, T. Vogt, and M. Bonnet, J. Solid State Chem. 84, 39 (1990).

[31] K. P. Belov and V. I. Sokolov, Usp. Fiz. Nauk 121, 285 (1977) [Sov. Phys. Usp. 20, 149 (1977)].

[32] M. Lahoubi, M. Guillot, A. Marchand, F. Tcheou, and E. Roudaut, IEEE Trans. Magn. 20, 1518 (1984).

[33] N. Hur, S. Park, S. Guha, A. Borissov, V. Kiryukhin, and S.-W. Cheong, Appl. Phys. Lett. 87, 042901 (2005).

[34] D. Louca, K. Kamazawa, and T. Proffen, Phys. Rev. B 80, 214406 (2009).

[35] P. D. Rogers, Y. J. Choi, E. C. Standard, T. D. Kang, K. H. Ahn, A. Dubroka, P. Marsik, C. Wang, C. Bernhard, S. Park, S.-W. Cheong, M. Kotelyanskii, and A. A. Sirenko, Phys. Rev. B 83, 174407 (2011).

[36] A. J. Sievers and M. Tinkham, Phys. Rev. 129, 1995 (1963).

[37] J. Kaplan and C. Kittel, J. Chem. Phys. 21, 760 (1953).

[38] T. D. Kang, E. Standard, K. H. Ahn, A. A. Sirenko, G. L. Carr, S. Park, Y. J. Choi, M. Ramazanoglu, V. Kiryukhin, and S.-W. Cheong, Phys. Rev. B 82, 014414 (2010).

[39] T. D. Kang, E. C. Standard, P. D. Rogers, K. H. Ahn, A. A. Sirenko, A. Dubroka, C. Bernhard, S. Park, Y. J. Choi, and S.-W. Cheong, Phys. Rev. B 86, 144112 (2012).

[40] T. N. Stanislavchuk, T. D. Kang, P. D. Rogers, E. C. Standard, R. Basistyy, A. M. Kotelyanskii, G. Nita, T. Zhou, G. L. Carr, M. Kotelyanskii, and A. A. Sirenko, Rev. Sci. Instrum. 84, 023901 (2013).

[41] M. Tinkham, Phys. Rev. 124, 311 (1961).

[42] See Supplemental Material at http://link.aps.org/ supplemental/10.1103/PhysRevLett.122.237401 for magnetic and optical properties of Dy iron garnet. 